\title{
A Novel Technique to Design Flat Fresnel Lens with Uniform Irradiance Distribution
}

\author{
Pham Thanh Tuan, Vu Ngoc Hai, Seoyong Shin* \\ Department of Information and Communication, Myongji University, Yongin City, Republic of Korea
}

Email address:

sshin@mju.ac.kr (S. Shin), pttuan1412@gmail.com (P. T. Tuan)

${ }^{*}$ Corresponding author

\section{To cite this article:}

Pham Thanh Tuan, Vu Ngoc Hai, Seoyong Shin. A Novel Technique to Design Flat Fresnel Lens with Uniform Irradiance Distribution. International Journal of Energy and Power Engineering. Vol. 5, No. 2, 2016, pp. 73-82. doi: 10.11648/j.ijepe.20160502.17

Received: February 28, 2016; Accepted: April 29, 2016; Published: May 5, 2016

\begin{abstract}
In this paper, we propose a novel technique to design flat Fresnel lens to achieve big concentration ratio, high uniform irradiance distribution while keeping F-number small using both refraction and total internal reflection (TIR) phenomena. Also, this method can be used to design concentrated photovoltaic (CPV) system without secondary optics lens (SOE). In this technique, Fresnel lens is constructed by many prisms, which are built by ideal Cartesian oval. The design process of Fresnel lens was performed using Matlab program. Ray tracing technique has been used to optimize the structure of lens using LightTools ${ }^{\mathrm{TM}}$ software. The simulation results have been shown that Fresnel lens has a good optical property such asconcentration ratio is $2500 \mathrm{x}$, F-number $=0.4$, and high uniform irradiance distribution.
\end{abstract}

Keywords: Fresnel Lens, CPV System, Total Internal Reflection, Second Optics Lens

\section{Introduction}

Nowadays, the advantages of photovoltaic technology help improve efficiency of solar cell significantly. The conversion efficiency of solar cell with $\mathrm{GaInP} / \mathrm{GaInAs} / \mathrm{Ge}$ multi-junction structure can be reached to $42 \%[1,2]$. However, the high cost of multi-junction cells leads to high price of solar systems. An effective way to reduce cost is to cut down the amount of required cell area while still increasing the cell efficiency by using concentrated photovoltaic system (CPV) [2, 3].

Using CPV systems is a good promising option to reduce cost and increase application ability in reality of photovoltaic systems. However, almost CPV systems still have some challenges such as non-uniform irradiance distribution, creating hot spot point over cell surface. Non-uniform irradiance and hot spot point degrade the reliability, the conversion efficiency, and life time of solar cell [1]. To solve these problems, CPV has been constructed with two lenses $[4,5]$. The first lens is Fresnel lens [6, 7, 8] which concentrates sunlight to a point onto second lens (SOE). The second lens which is close to cell surface has been used to redistribute sunlight uniformly over solar cell surface, which makes the design process become more complex [9]. To overcome this problem, there have been some efforts to design CPV system without second lens [1], [10]. However, these methods still have some limits in terms of increasing concentration ratio.

The uniformity and concentration ratio are two crucial parameters in designing Fresnel lens for CPV system in two reasons. First, the high uniformity of concentrated beam can increase conversion efficiency in multi-junction solar cell [11]. Second, the cost of solar system can be reduced if CPV with high concentration ratio is used. In this paper, we propose a novel idea to design CPV system with uniform irradiance distribution using flat Fresnel lens without SOE. We also propound a new technique to increase concentration ratio of CPV while keeping F-number small. The Fresnel lens shape can be built in 3D by using Solid Works and LightTools $^{\mathrm{TM}}$ software. The optical performances of the designed Fresnel lens are simulated by using the nonsequential ray tracing software, LightTools ${ }^{\mathrm{TM}}$. An optimized structure is designed by modulating the geometric parameters of the Fresnel lens. The concentration ratio of $2500 x, F-$ number of 0.4 , and high uniformity were achieved in the optimized design of Fresnel lens. 


\section{Basic Ideas}

The optics systems applied to CPV require large area, light weight, mass production, and low image quality to reduce cost of photovoltaic systems. So, Fresnel lens is a best choice and it has been used widely in CPV. Typical structure of Fresnel lens consists of series of concentric grooves which act as individual refracting surfaces, bending parallel light rays to a common focal point. Figure 1(a) shows a traditional Fresnel lens. By the nature of optical property of conventional Fresnel lens, irregular illumination on solar cell is non-uniform, which produces a local hot point on cell surface. At that hot point, the temperature is so high that it affected Ohmic contact, which degraded conversion efficiency and life time of solar cell significantly. The decrease of conversion efficiency due to non-uniform irradiance can be compensated partly by using unique structure solar cell. That is achieved by connecting solar cell units in serially to match the voltage level at each sub region. Then these sub regions with equal voltage level are connected in parallel to collect the electric current from the cell assembly [10]. However, that is not really a fundamental solution to solve these challenges of solar cell system.

To overcome disadvantages of conventional Fresnel lens we propose a novel technique to design Fresnel lens. The basic idea of the Fresnel lens for the 2-D CPV system is depicted in Figure 1(b). Each groove of Fresnel lens focus bundle of parallel rays to focal point $\mathrm{P}_{\mathrm{ng}}$, then the bundle of light has been distributed over receiver with uniform irradiance.

In our design, the process consists of four steps described as follows. That is shown in Figure 1(b).

- Step 1: The light which comes to extreme left position of groove $\mathrm{P}_{\mathrm{s}}$ will be refracted and directed to the extreme right position of receiver.

- Step 2: The light which comes to extreme right position of groove $\mathrm{P}_{\mathrm{n}}$ will be refracted and directed to the extreme left position of receiver.

- Step 3: The light at $P_{n}$ and $P_{s}$ position will be focused at $\mathrm{P}_{\mathrm{ng}}$ which is in-between lens and receiver. The rest of bundle rays coming to groove between $P_{n}$ and $P_{s}$ will be refracted and directed to $\mathrm{P}_{\mathrm{ng}}$, then distributed uniformly over receiver.

- Step 4: The same procedure is repeated for every groove. One discontinuity in the normal of exit surface at $P_{s}$ and $P_{n}$ is necessary. That helps lens shape becomes continuous.

Every bundle of rays coming to groove will be distributed over cell surface with uniform irradiance; therefore, light coming to Fresnel lens will be distributed uniformly over cell surface.

The advantage of this design is that we can distribute light uniformly over the receiver with any size. However, there is a challenge of finding out $\mathrm{P}_{\mathrm{ng}}$ position accurately. If the position of $\mathrm{P}_{\mathrm{ng}}$ is not accurate then distribution irradiance will not be uniform over the receiver. Some different positions of $\mathrm{P}_{\mathrm{ng}}$ and irradiance distributions are shown respectively in Figure 2.
Fortunately, we can overcome the challenge of finding $\mathrm{P}_{\mathrm{ng}}$ position by using computer programming. Nowadays, computer technology has been developed powerfully. Computer and computation program can solve millions of calculations per second. Thus, finding the position of $\mathrm{P}_{\mathrm{ng}}$ accurately is not impossible. More accurate position of $\mathrm{P}_{\mathrm{ng}}$ requires more time. The schematic of algorithm of designing the lens and finding $P_{n g}$ position is shown in figure 3.

Every groove of lens needs two surfaces to distribute light uniformly over receiver. First is entry surface which is perpendicular with bundle of parallel rays and second is exit surface which is a Cartesian oval surface. The light coming to entry surface will exit at exit surface and become focused at $\mathrm{P}_{\mathrm{ng}}$ position. Every light must have the same optical path length (OPL) satisfying following equation.

$$
\begin{aligned}
& a_{1}=n \times a_{2}+a_{2}^{\prime}=n \times a_{3}+a_{3}^{\prime}=\ldots \\
& \ldots=n \times a_{m}+a_{m}^{\prime}=n \times a_{\text {end }}+a_{\text {end }}^{\prime}=O P L
\end{aligned}
$$

Where $\mathrm{n}$ is refractive index. $\mathrm{a}$ and a' are path lengths of a ray in medium (Figure 4). From equation (1) we can calculate the small pieces of exit surface. These small pieces correspond to Cartesian ovals (see Figure 5 (a)), and in this way, we can build whole flat Fresnel lens.

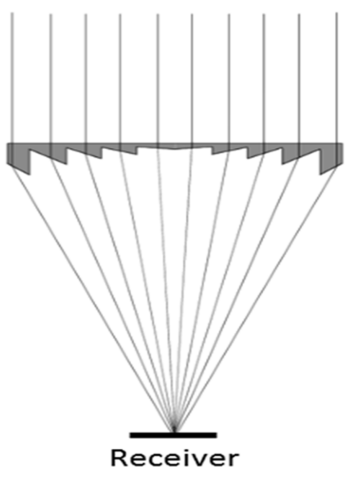

(a)

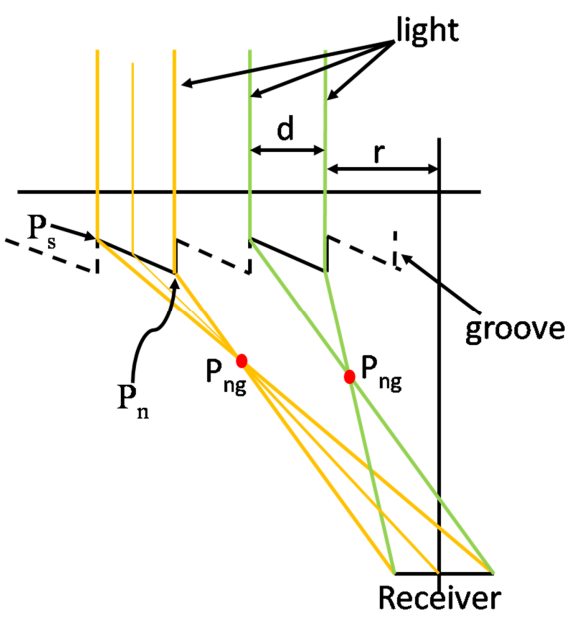

(b)

Figure 1. The difference between a) Conventional Fresnel lens [12] and b) Newly designed Fresnel lens. 


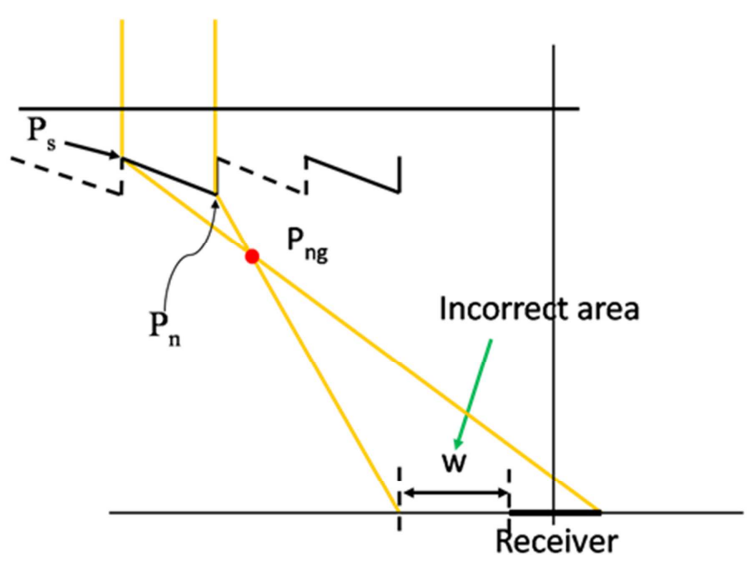

(a)

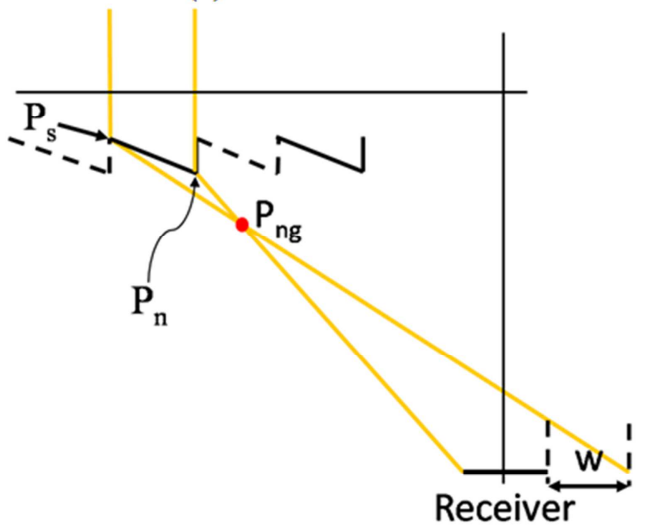

(c)

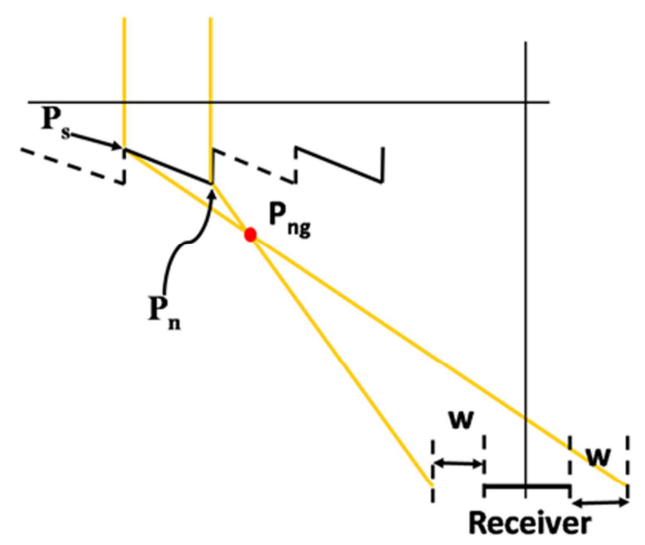

(b)

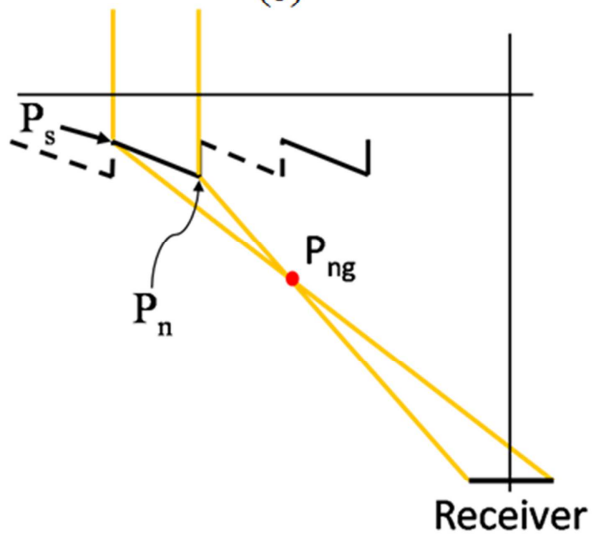

(d)

Figure 2. Some different positions of $P_{n g}$ and distribution irradiance over receiver: a) irradiance distribution shifted to left of receiver, b) irradiance distribution shifted to both sides of receiver, $c$ ) irradiance distribution shifted to right of receiver, d) irradiance distribution is exactly over receiver.

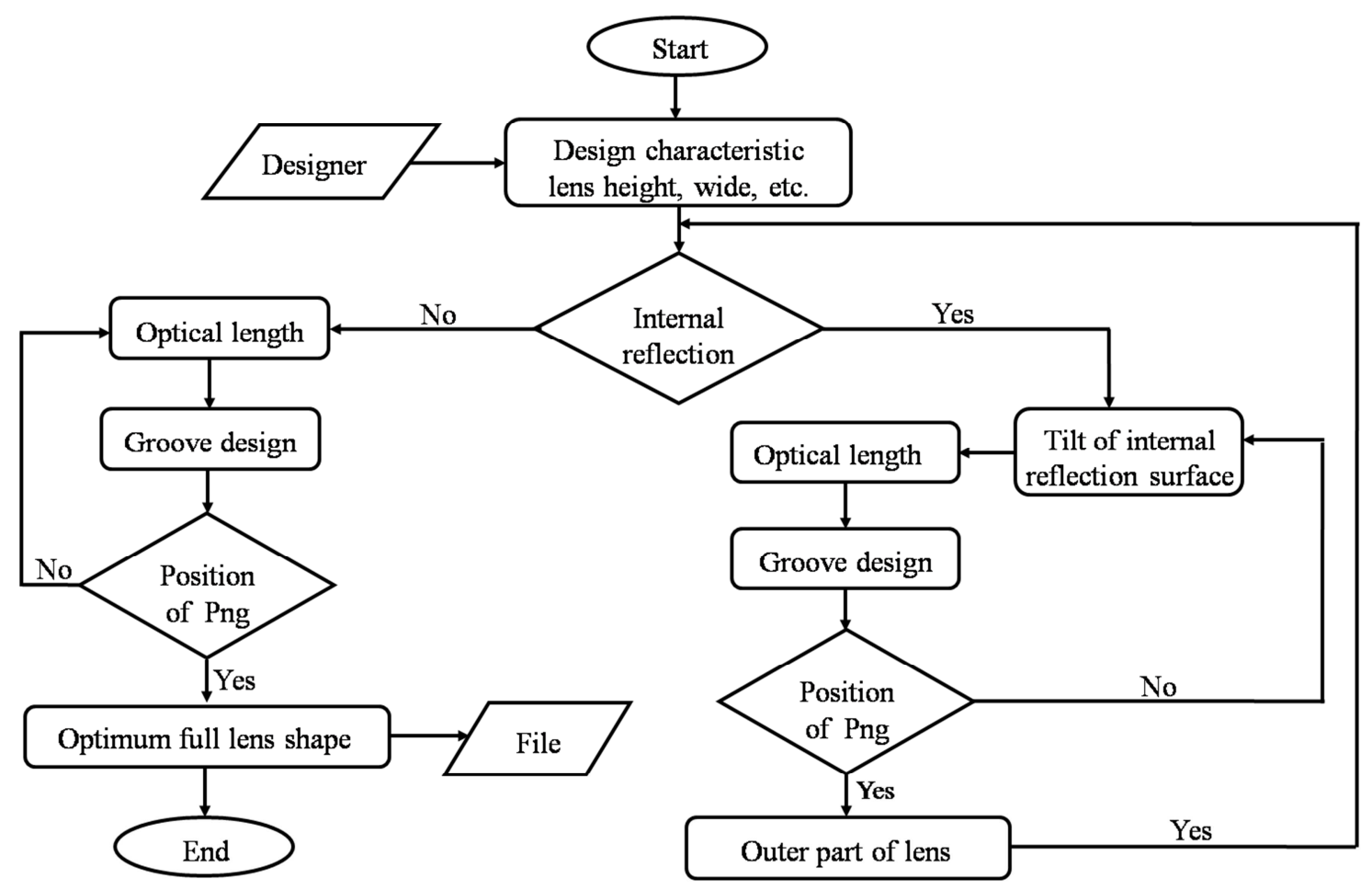

Figure 3. Flow chart of optimum lens calculation. 

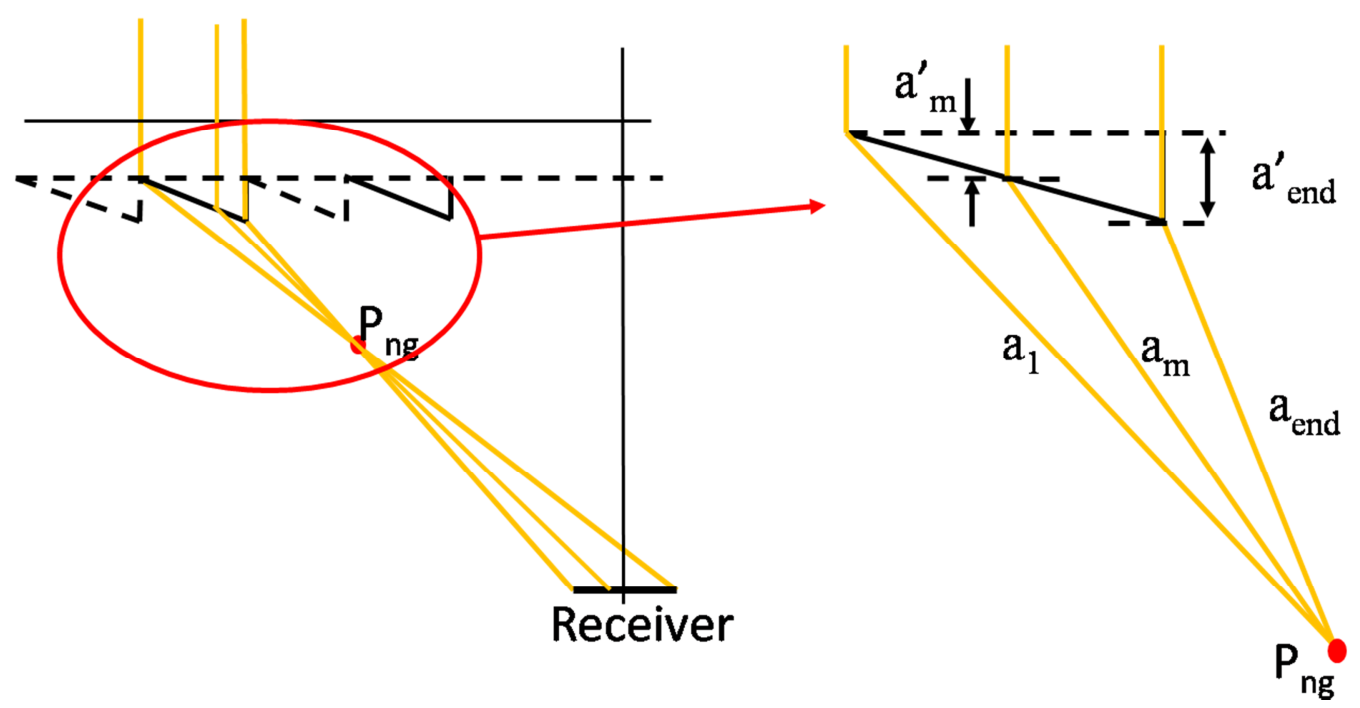

Figure 4. Optical path length of ray in groove.

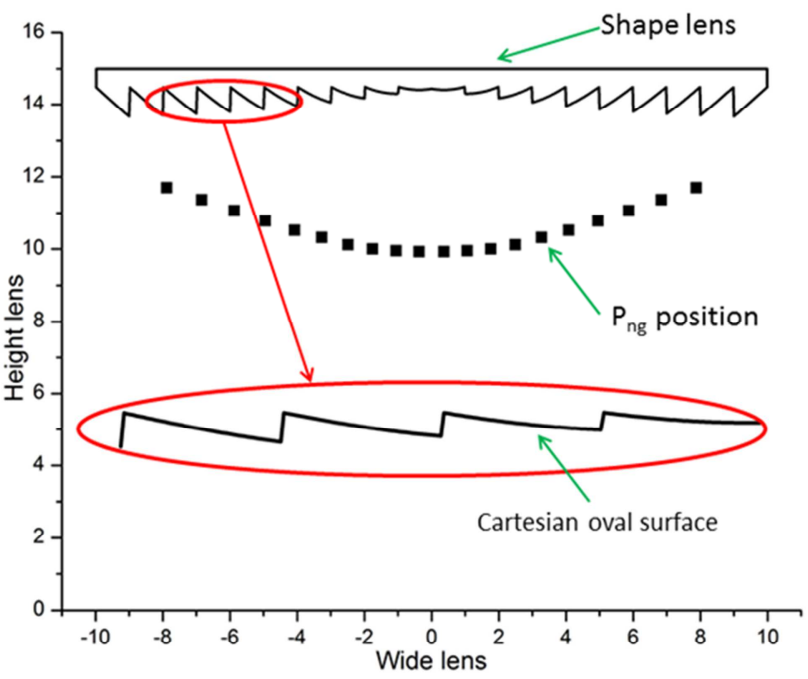

(a)

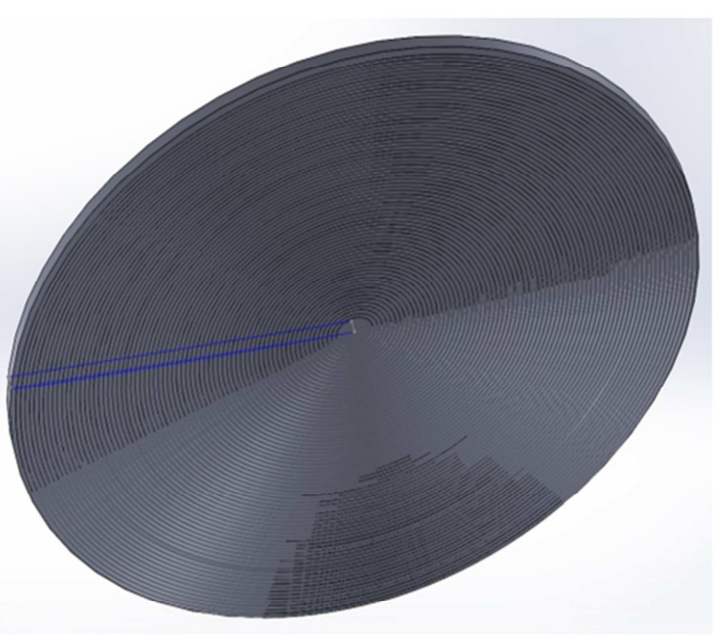

(b)

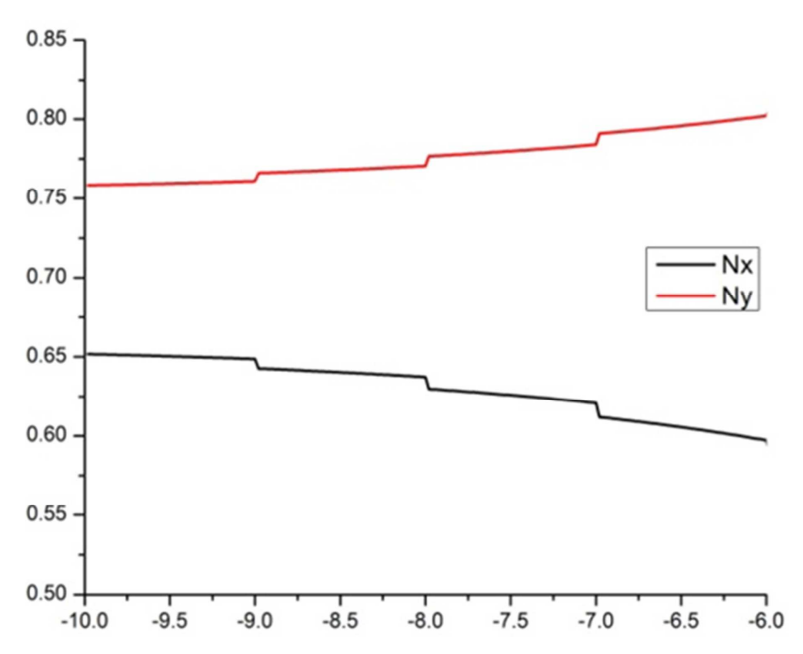

(c)

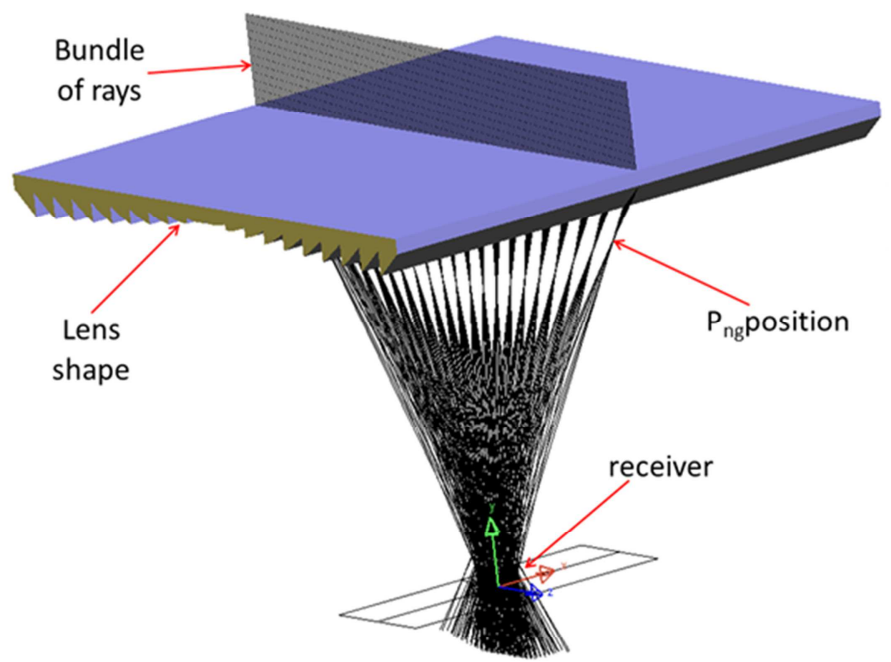

(d)

Figure 5. Shape and feature of flat Fresnel lens. a) Lens shape, $P_{n g}$ position, and exit surface as Cartesian oval in 2-D. b) Circular Fresnel lens in 3-D. c) The two components $\left(N_{x}, N_{y}\right)$ of the normal vector of the exit surface. d) Square Fresnel lens shape, $P_{n g}$ position in 3-D. 
After design process, flat Fresnel lens has a shape as shown in Figure 5 (a). The flat Fresnel lens has been designed with large groove to show the profile clearly. Figure 5 (a) also shows exit surface shape of Cartesian oval and $P_{n g}$ positions. In addition, the normal vector of Cartesian changed through exit surface and it can be calculated by two component $\mathrm{N}_{\mathrm{x}}$ (follow $\mathrm{x}$ direction) and $\mathrm{N}_{\mathrm{y}}$ (follow $\mathrm{y}$ direction). Figure 5 (c) shown the volume of Cartesian normal vector with two components $\mathrm{N}_{\mathrm{x}}$ and $\mathrm{N}_{\mathrm{y}}$. In this design method, the discontinuity of normal vector at each groove edge is needed for the shape of lens continuity. Moreover, the lens with square or circle shape in 3-D can be built by using Solidworks and LightTools ${ }^{\mathrm{TM}}$ software and was shown in Figure 5 (b) and (d).

\section{Increasing Dimension of Lens, Loss Problem, and a Solution}

Lens designers are intending to increase concentration ratio as big as possible while keeping F-number small. In this part, we introduce a new technique to increase concentration ratio and decrease the optics loss.

In our design, the exit surfaces of lens are Cartesian ovals. Dimension of flat Fresnel lens will be increased until total internal reflection (TIR) appears. That is shown in Figure 6. If the TIR happens, the bundle of parallel rays remains inside the lens and will not reach to the receiver.

TIR phenomenon is really a challenge to increase dimension of Fresnel lens. Fortunately, we can solve this problem by changing the shape of groove in Fresnel lens. Some techniques have been used to avoid TIR inside the lens [4], [13]. In [4], exit surface consists of two tilt surfaces where total internal reflection happens. This design, the loss appears at extreme right position of exit surface which shown in Figure 7 (a). In [13], whereas, the TIR repeats two times at output surface, however, this leads to the calculation become more complex. To decrease loss and increase dimension of Fresnel lens, we propose a new technique in the design of lens as shown in Figure 7(b).

In this design, the groove of Fresnel lens consists of flat surface S1 and Cartesian oval surface S2. The bundle of parallel rays will be reflected (TIR) at S1 surface, and become refracted at $\mathrm{S} 2$ surface, then the rays will be focused on $\mathrm{P}_{\text {ng }}$ position and distributed over the receiver (see Figure 7 (b)).

Figure 8 shows some grooves designed by using TIR phenomenon. If the tilting angle of S1 surface is $\alpha=45^{\circ}$, the TIR ray will be refracted 90 degrees. In this design, S2 surface is too close to S1 surface of next groove (Figure 8). Therefore, the bundle of rays exit from S2 surface will be refracted again at S1 surface of next groove, which makes the rays not reach to the receiver.

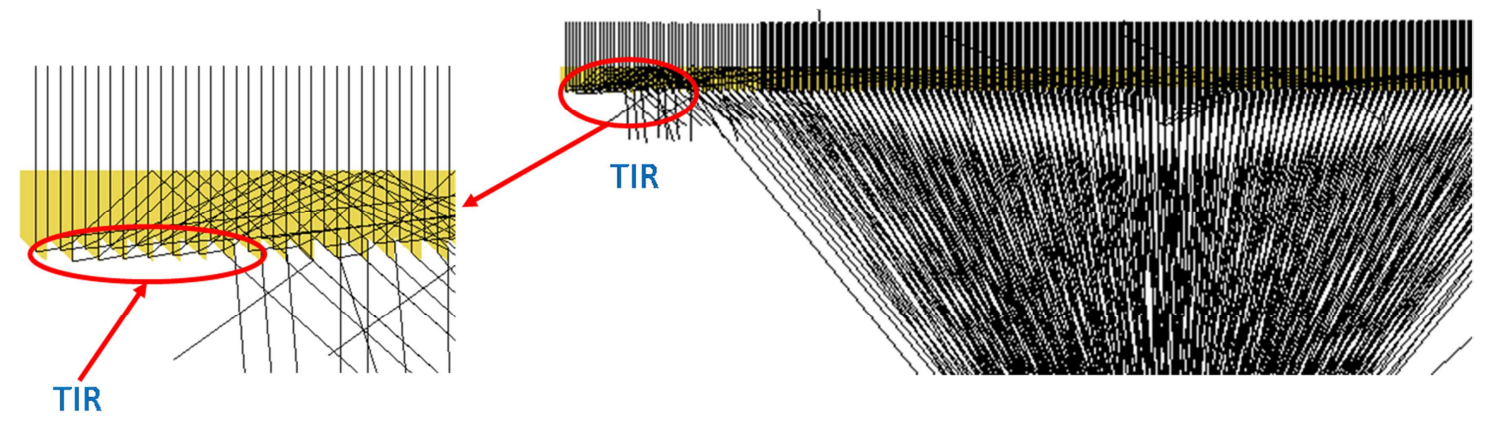

Figure 6. Total internal reflection appears at positions indicated.

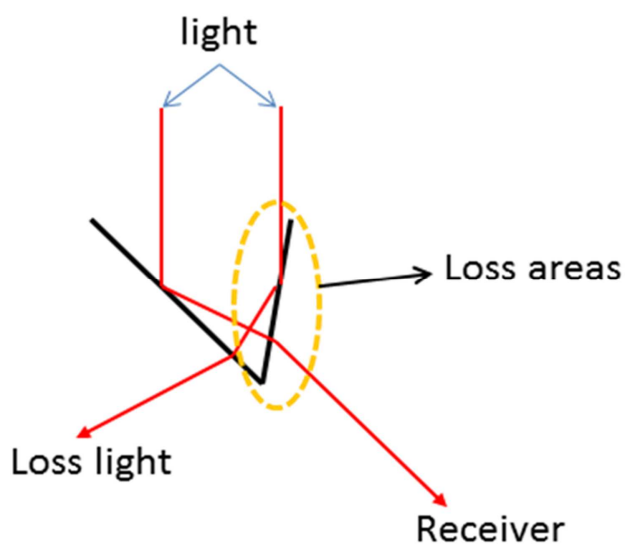

(a)

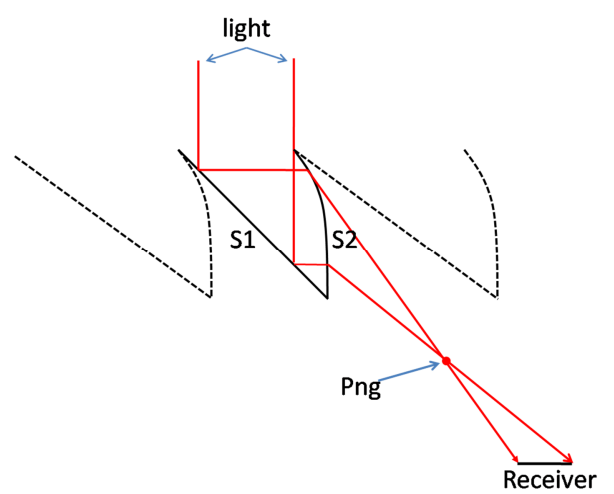

(b)

Figure 7. Schematic of groove design using TIR a) Conventional groove [4] and b) our groove design (the picture is not scale). 


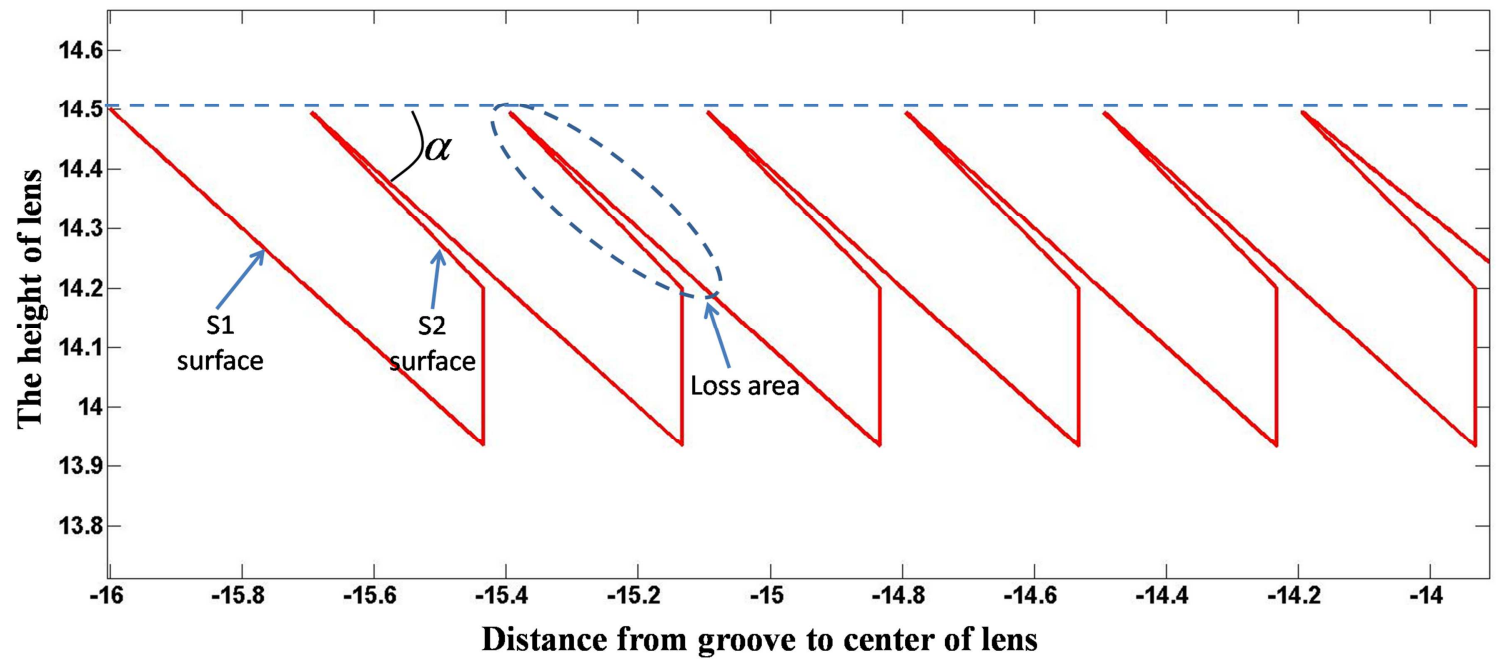

Figure 8. The grooves of flat Fresnel lens have been designed with the tilt of S1 surface is $\alpha=45^{\circ}$.

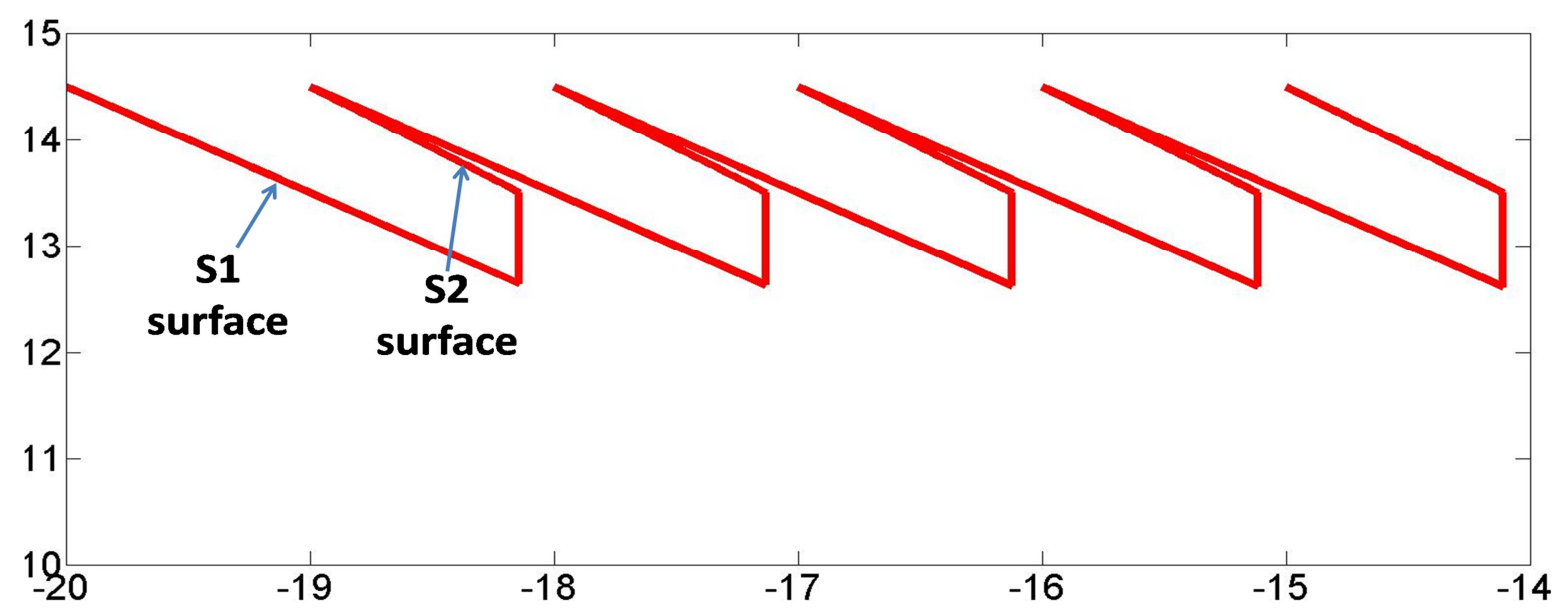

(a)

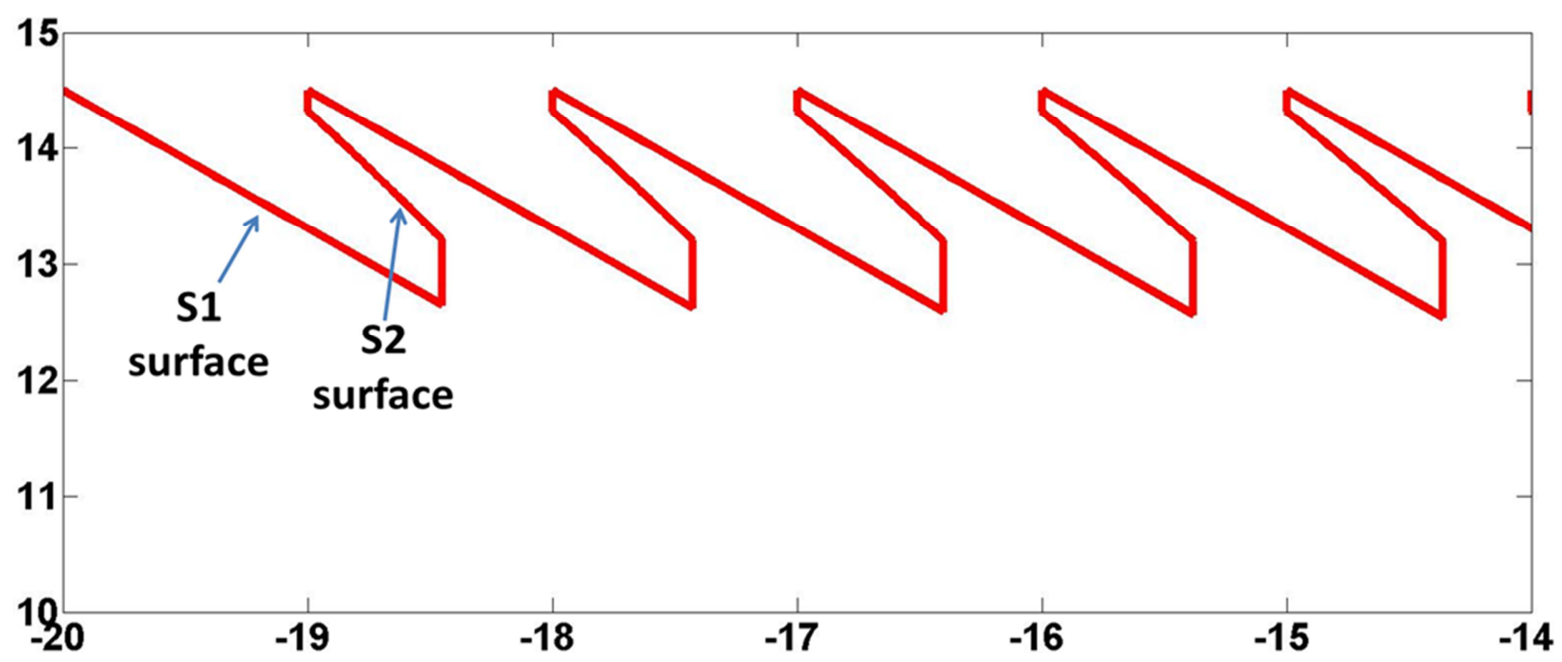

(b) 


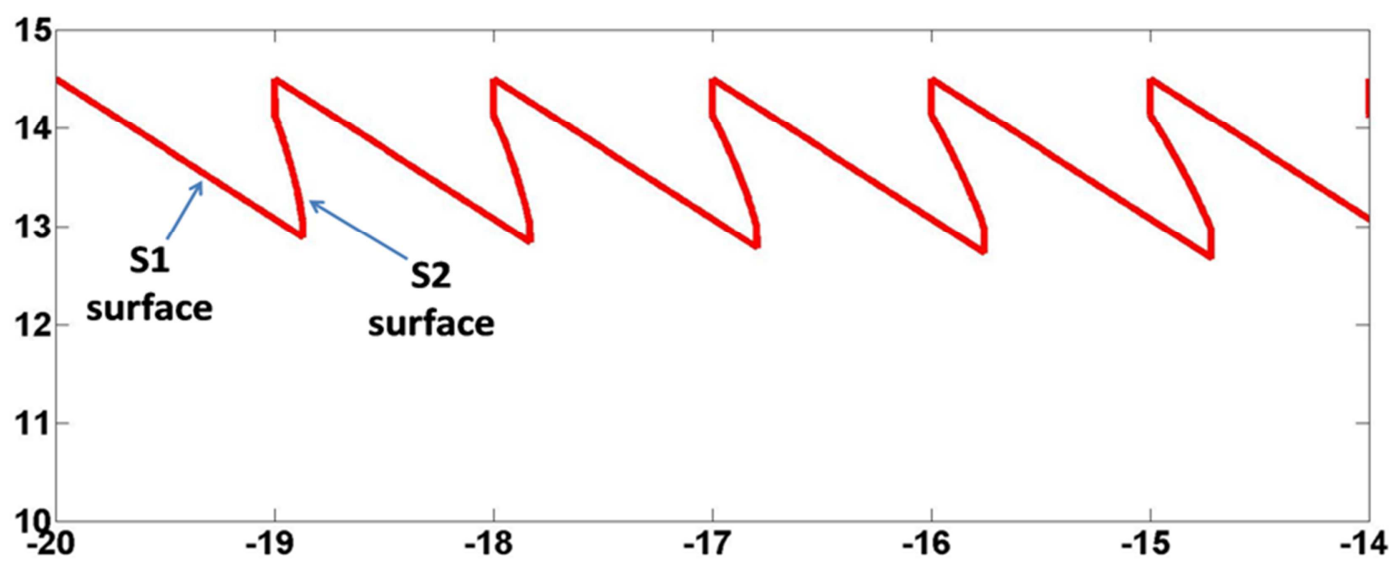

(c)

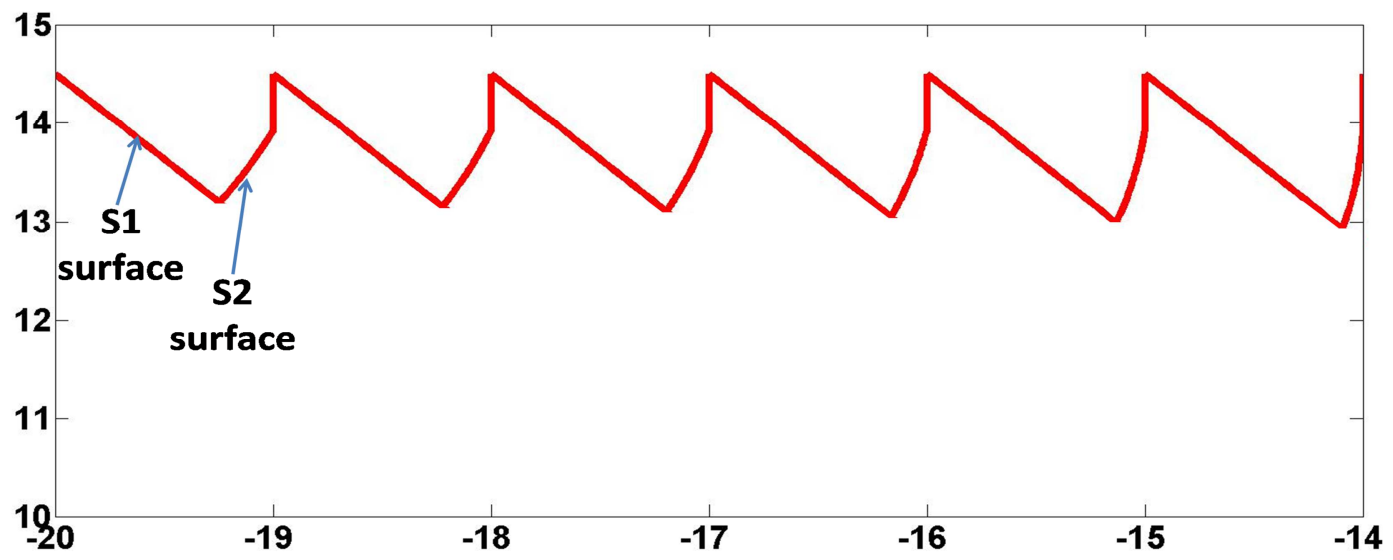

(d)

Figure 9. Groove shape (exit surface) of Fresnel lens with different tilt angles of S1 surface a) $\alpha=45^{\circ} \mathrm{b}$ ) $\alpha=50^{\circ} \mathrm{c}$ ) $\alpha=55^{\circ} \mathrm{d}$ ) $\alpha=60^{\circ}$.

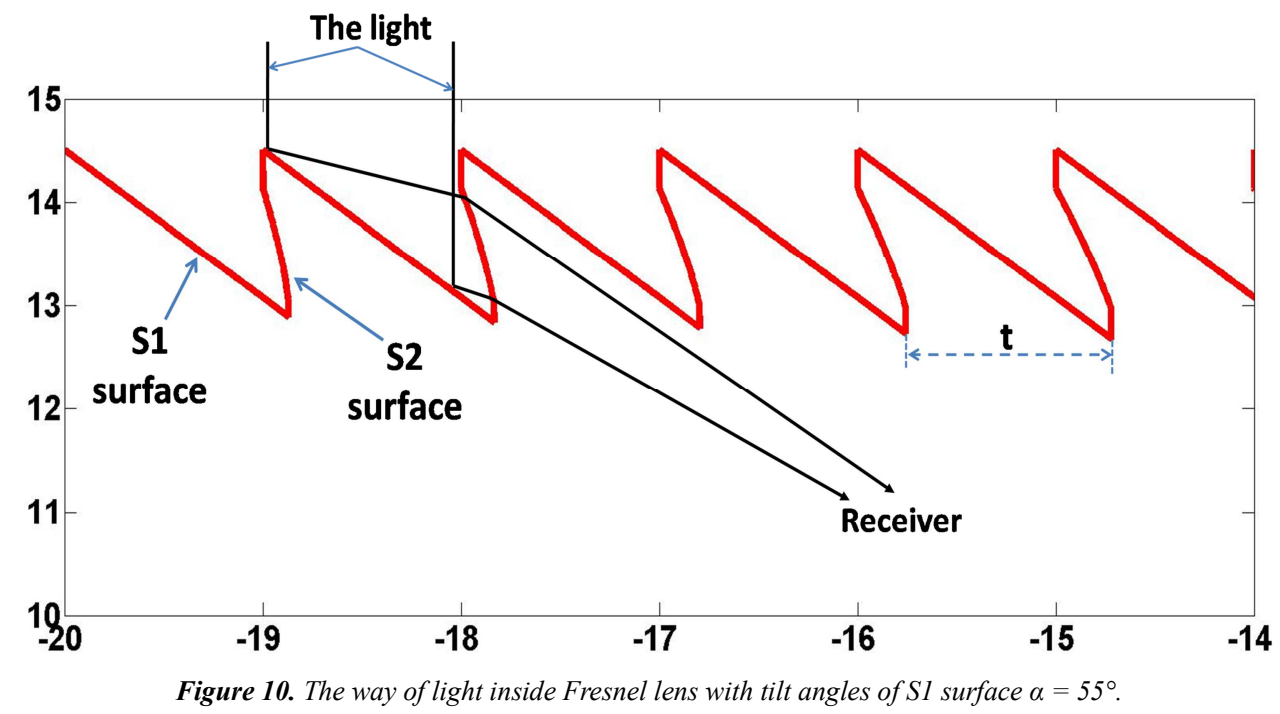

Consequently, the tilt of S1 surface has been investigated to decrease optics loss at loss area (see Figure 8). Figure 9 shows the tilt of $\mathrm{S} 1$ surface at different angles $\left(\alpha=45^{\circ}, 50^{\circ}\right.$, $55^{\circ}, 60^{\circ}$ ). Fresnel lens with tilt angle S1 $\alpha=55^{\circ}$ is the least loss comparing with the rest because of two reasons. First reason: distance $t$ (see figure 10) is big enough for the rays to exit from S2 surface and come to receiver without being refracted at S1 surface of next groove. Second reason: S2 surface is tilted forward to the right direction, as a result, there is no rays to be internally reflected at $\mathrm{S} 2$ surface.

However, there still exits loss at the tip of groove. The lights coming from the upper position of S2 surface of the groove were interrupted by the surface (S1) of next groove (see Figure 11(a)). We can decrease optics loss more by 
changing the sharp of groove. The tip of groove has been cut (see Figures 11(b) and (d)). That helps the light come to the receiver easier.

In brief, we introduced a new technique to increase dimension of Fresnel lens and decrease optics loss in the design process by using TIR.

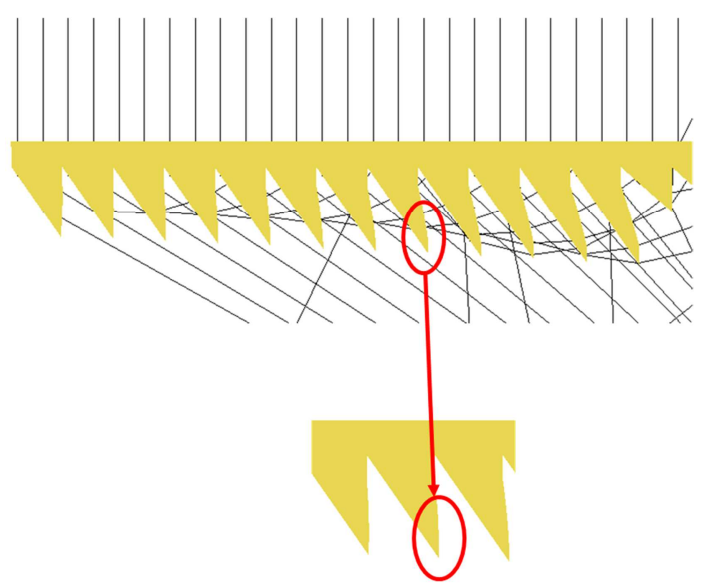

(a)

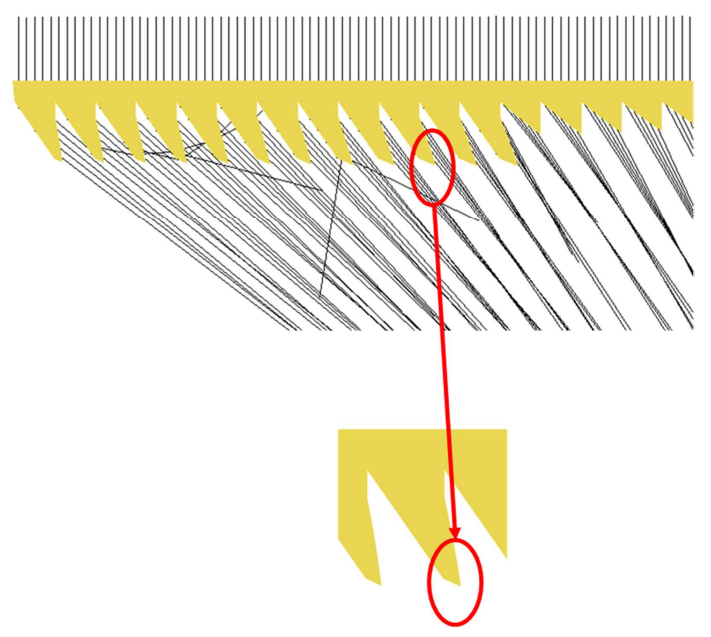

(b)

\section{Performance and Discussion}

In our design, the flat Fresnel lens has diameter $L=50 \mathrm{~cm}$. It concentrates and distributes light over receiver with diameter $l=1 \mathrm{~cm}, 2 \mathrm{~cm}$, and $3 \mathrm{~cm}$, alternately. With the definition of the geometrical concentration $C=(L / l)^{2}$, the concentration ratios becomes $2500 \mathrm{x}, 1100 \mathrm{x}$, and $625 \mathrm{x}$ sun, alternately. The irradiance distributions at the cell lens are estimated to be high uniform. Lens has height $20 \mathrm{~cm}$ and F-number 0.4 (height / diameter of lens).

Simulated process was performed by LightTools $^{\mathrm{TM}}$ software. Simulation parameters are following: power of light source is 2000 Watt, the number of rays viewed in ray tracing program is 400 . In addition, the ray tracing technique has been used to characterize lens' performance.

Figure 12 shows the race tracing results of flat Fresnel lens. Figure 12 (a) is the result without TIR based design of groove.In this structure, the diameter of effective area of Fresnel lens where the light becomes concentrated at the receiver is just only $28 \mathrm{~cm}$. Figure 12 (b) shows the result with TIR based design of groove. The diameter of lens becomes increased to $50 \mathrm{~cm}$, which means the whole lens contributes to the concentration of light perfectly.

Figure 13 shows the way of rays exits from flat Fresnel lens. At outer areas, the light exits the lens at right side of groove (S2 surface) while the light exits the lens at left side of groove at inner areas (S1 surface). This is because we used refracting phenomenon to design inner groove, whereas we used total internal reflecting phenomenon to design outer groove of lens (told in third part). The interface between inner and outer area can be estimated by solving mathematics. Refracted angle will be increased if radius of lens increases. When it reaches $90^{\circ}$, the total internal reflection appears then the position of interface will be determined. The inner area has been limited by TIR, whereas the size of outer doesn't have a limit clearly. The limit of outer area depends on designer and efficiency of optical loss. The simulation process help designer decide how big lens should be.

Figure 11. The shape of groove a) the sharp groove and ray tracing, b) the cutting groove and ray tracing.

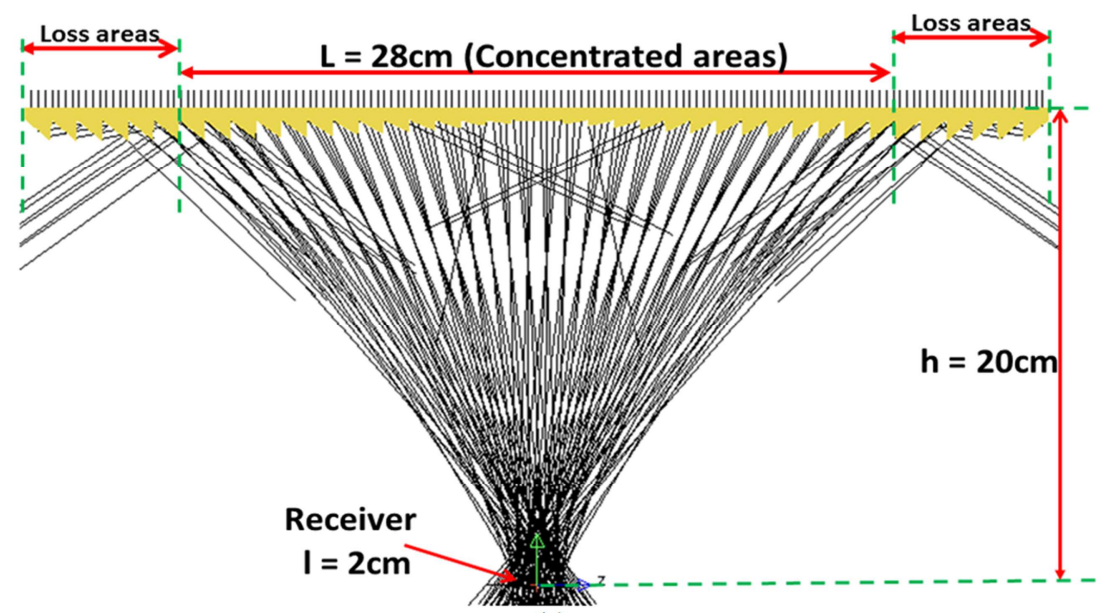

(a) 


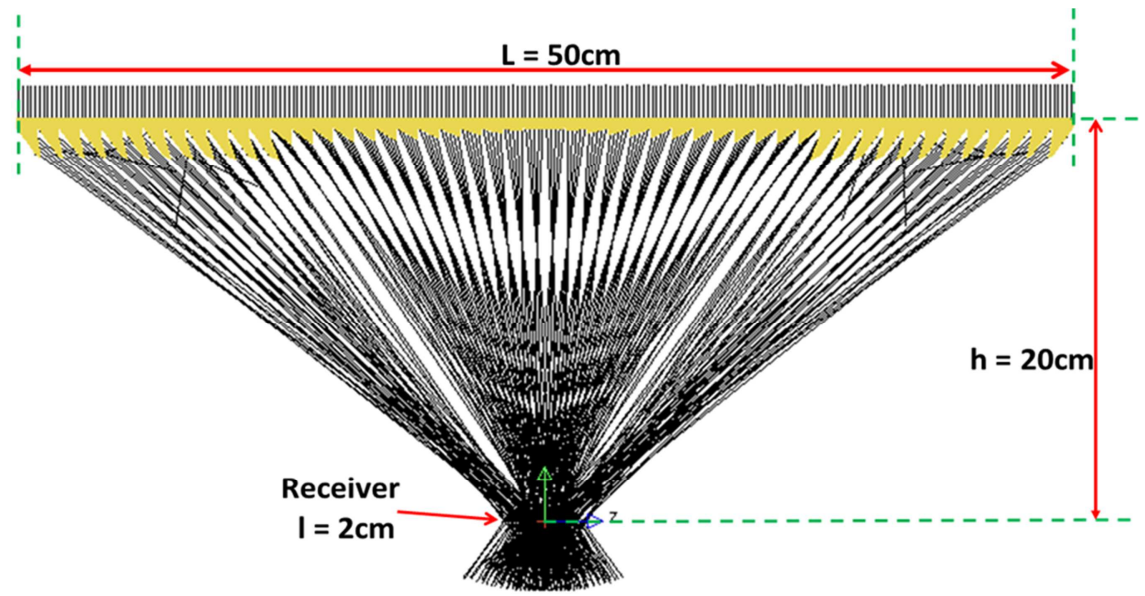

(b)

Figure 12. The flat Fresnel lens a) not using TIR and b) using TIR to design groove.

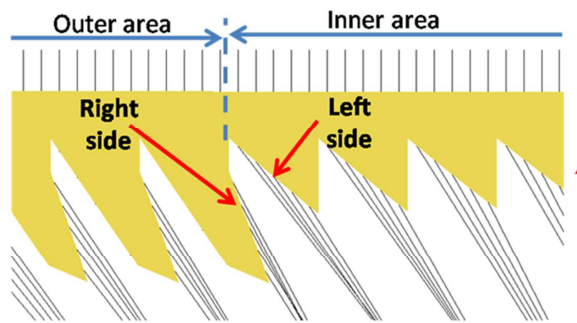

(a)

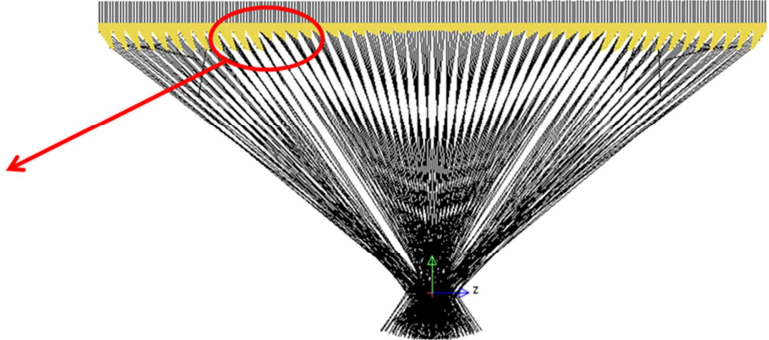

(b)

Figure 13. The ray tracing of a) outer and inner areas of flat Fresnel lens and b) whole lens.

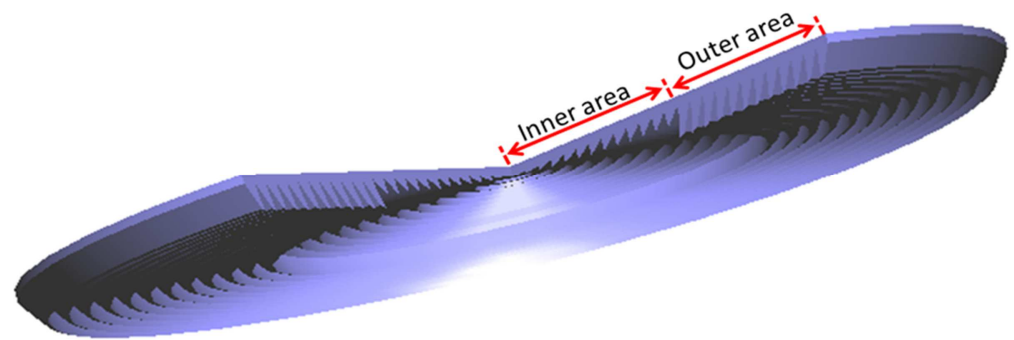

Figure 14. The flat Fresnel lens in 3-D after finishing design process

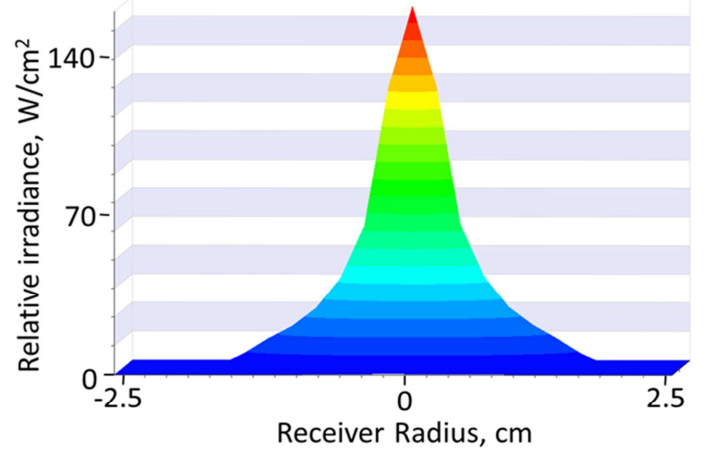

(a)

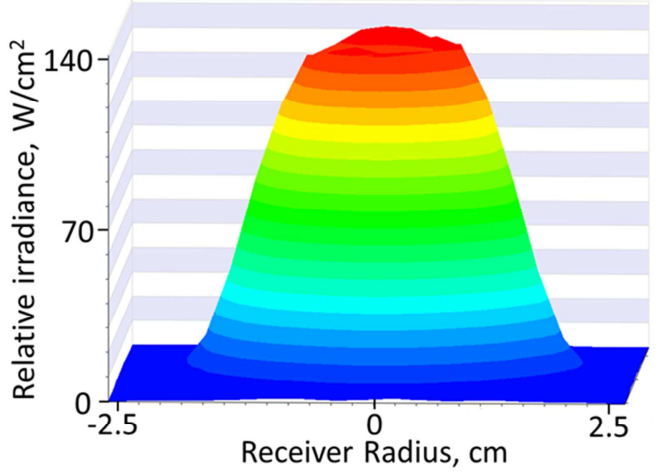

(b)

Figure 15. Distribution of irradiance over the receiver: a) conventional Fresnel lens and b) our designed Fresnel lens.

Figure 14 shows the flat Fresnel lens shape in 3-D. Lens shape consists of two parts: inner and outer areas. Inner area designed by using refraction phenomenon has been constructed by some smaller grooves. Meanwhile, outer area designed by using TIR has been constructed by some bigger grooves. 
Furthermore, we have investigated the uniformity of irradiance distribution over receiver by using simulation in LightTools $^{\mathrm{TM}}$. The Fresnel lens collects sun light and distributes it over the receiver which has diameter $3 \mathrm{~mm}$. The uniformity has been described in Figure 15 (b). In this figure,the irradiance distribution over the receiver in our designed Fresnel lens is shown. It is quite uniform. There is no any hot spot over the receiver area, whereas, the conventional Fresnel lens has a hot spot in the center of lens.

\section{Conclusions}

A novel technique to design flat Fresnel lens for CPV system was presented to increase the concentration ratio, reduce optical loss, and improve uniformity of irradiance distribution over receiver. The simulation results have been identified that using our method to design Fresnel lens the concentration ratio can achieve $2500 \mathrm{x}$ while $\mathrm{F}$-number keeping small (F-number $=0.4$ ). In addition, we can use this technique to design CPV system without SOE. Also, Fresnel lens designed by our technique collects and distributes uniform sunlight over receiver with any size. All these factors will be helpful to enhance the performance of CPV and reduce the cost of solar systemsof multi-junction solar cells.

\section{Acknowledgements}

This work was supported by a National Research Foundation of Korea (NRF) grant funded by the Korea government (MSIP) (No. 2014R1A2A1A11051888).

\section{References}

[1] Jui-Wen Pan, Jiun-Yang Huang, Chih-Ming Wang, Hwen-Fen Hong, Yi-Ping Liang, "High concentration and homogenized Fresnel lens without secondary optics element," Optics Communications, 2011, vol. 284, pp. 4284-4288.

[2] Mehrdad Khamooshi, Hana Salati, Fuat Egelioglu, Ali Hooshyar Faghiri, Judy Tarabishi, and Saeed Babadi, "A Review of Solar Photovoltaic Concentrators," International Journal of Photoenergy, 2014, vol. 2014, article ID 958521.
[3] E. Lorenzo and A. Luque, "Comparison of Fresnel lenses and parabolic mirrors as solar energy concentrators," Applied Optics, 1982, vol. 21, pp. 1851-1853.

[4] Lei Jing, Hua Liu, Hui-fu Zhao, Zhenwu Lu, Hongsheng Wu, He Wang, and Jialin Xu, "Design of novel compound Fresnel lens for High-performance photovoltaic concentrator," International Journal of Photoenergy, 2011, vol. 2012, article ID 630692 .

[5] Stanislas Sanfo, Abdoulaye Ouedraogo, "Contribution to the Optical Design of A Concentrator with Uniform Flux for Photovoltaic Panels," Advances in Energy and Power, 2015, vol. 3, pp. 82-89.

[6] Ralf Leutz, Akio Suzuki, Atsushi Akisawa, Takao Kashiwagi "Design of A Nonimaging Fresnel Lens for Solar Concentrators," Solar Energy, 1999, Vol. 65, No. 6, pp. 379387.

[7] Juan C. González, "Design and analysis of a curved cylindrical Fresnel lens that produces high irradiance uniformity on the solar cell," Applied Optics, 2009, Vol. 48, No. 11 , pp. 2127-2132.

[8] N. Yamada and T. Nishikawa, "Evolutionary algorithm for optimization of nonimaging Fresnel lens geometry," Optics Express, 2010, Vol. 18.

[9] Juan C. Minano, Pablo Benitez, Pablo Zamora, Marina Buljan, Ruben Mohedano, and Asuncion Santamaria, "Free-form optics for Fresnel lens based photovoltaic concentrators" Optical Society of America, 2013, vol. 21, No. S3.

[10] Kwangsun Ryu, Jin-Geun Rhee, Kang-Min Park, Jeong Kim, "Concept and design of modular Fresnel lenses for concentration solar PV system," Solar energy, 2006, vol. 80, pp. 1580-1587.

[11] E. A. Katz, J. M. Gordon, D. Feuermann, "Effects of ultrahigh flux and intensity distribution in multi-junction solar cells," Prog. Photovoltaics, 2006, vol. 14, pp. 297-303.

[12] http://www.fresneltech.com/.

[13] Ian Wallhead, Teresa Molina Jiménez, Jose Vicente García Ortiz, Ignacio Gonzalez Toledo, and Cristóbal Gonzalez Toledo, "Design of an efficient Fresnel-type lens utilizing double total internal reflection for solar energy collection," Optics express, 2012, vol. 20, No. S6. 